\title{
Comparativo entre a técnica convencional pós-exodontia de terceiros molares e o uso de Fibrina Rica em Plaquetas associado à ozonioterapia: relato de caso
}

Comparison between the conventional technique after third molar extraction and the use of fibrinrich plasma associated with ozone therapy: case report

Comparación entre la técnica convencional tras la extracción del tercer molar y el uso de plasma rico en fibrina asociado a la ozonoterapia: relato de caso

\author{
Stefani Almeida Gonçalves \\ ORCID: https://orcid.org/0000-0001-6950-2533 \\ Faculdade Patos de Minas, Brasil \\ E-mail: stefaanialmeida@hotmail.com \\ Thaynná Alves Dias Teodoro \\ ORCID: https://orcid.org/0000-0002-3883-4794 \\ Faculdade Patos de Minas, Brasil \\ E-mail: thaynna_alves_@hotmail.com \\ Érika Thamires de Oliveira \\ ORCID: https://orcid.org/0000-0002-3729-8440 \\ Faculdade Patos de Minas, Brasil \\ E-mail: erika88218788@gmail.com \\ Cláudia Maria de Oliveira Andrade \\ ORCID: https://orcid.org/0000-0003-4529-8106 \\ Faculdade Patos de Minas, Brasil \\ E-mail: claudia.andrade@faculdadepatosdeminas.edu.br \\ Lia Dietrich \\ ORCID: https://orcid.org/0000-0001-7887-8591 \\ Universidade Federal dos Vales do Jequitinhonha e Mucuri, Brasil \\ E-mail: lia.dietrich@ufvjm.edu.br \\ Marcelo Dias Moreira de Assis Costa \\ ORCID: https://orcid.org/0000-0001-9148-3674 \\ Universidade Federal de Uberlândia, Brasil \\ E-mail: marcelodmac@yahoo.com.br
}

\begin{abstract}
Resumo
Os terceiros molares normalmente são os últimos dentes a irromper na cavidade bucal, na qual evidência ausências de espaços nas arcadas dentárias, levando-os comumente a serem encontrados em posição como inclusos e/ou impactados. Nesses casos são frequentes algumas complicações pós-operatória com presença de sinais e sintomas, tais como dor, edema, trismo, alveolite, hematomas locais e sangramentos. Esse trabalho tem como objetivo relatar um caso na literatura de tratamentos complementares com Fibrina Rica em Plaquetas (PRF) e óleo ozonizado na extração de terceiros molares comparado com a técnica convencional. Foi realizado uma busca de artigos científicos nas bases de dados PubMed e SciELO para embasamento científico e construção do relato de caso clínico. Paciente do sexo masculino, 20 anos de idade, com queixa principal de sintomatologia dolorosa nos terceiros molares inferiores. Perante anamnese, exame clínico e radiográfico foi indicado a extração dos dentes 38 e 48 semi-inclusos e proposto o tratamento complementar com a técnica de Fibrina Rica em Plaquetas (PRF) e uso tópico do óleo ozonizado pós exodontia no alvéolo 48 comparado com a técnica convencional no alvéolo 38. Foi observado melhoras sintomatológicas e clínicas na região do 48 comparado com a região do 38 após 7 dias de cirurgia. A Fibrina Rica em Plaquetas associado com uso tópico do óleo ozonizado mostrou-se eficaz neste caso de sinais e sintomas pós cirúrgico e novos estudos devem ser realizados para a criação de um protocolo clínico de tratamento.
\end{abstract}

Palavras-chave: Ozônio; Cicatrização; Fibrina rica em plaquetas; Pós-operatório.

\section{Abstract}

Third molars are usually the last teeth to erupt into the oral cavity, in which there is evidence of an absence of spaces in the dental arches, commonly leading them to be found in position as impacted and/or impacted. In these cases, some postoperative complications are frequent, with the presence of signs and symptoms, such as pain, edema, trismus, alveolitis, local bruises and bleeding. This paper aims to report a case in the literature of complementary treatments with platelet-rich fibrin (PRF) and ozonized oil in the extraction of third molars compared with the 
conventional technique. A search for scientific articles was performed in the PubMed and SciELO databases for scientific basis and construction of the clinical case report. Male patient, 20 years old, with chief complaint of painful symptoms in the lower third molars. Upon anamnesis, clinical and radiographic examination, extraction of teeth 38 and 48 semi-enclosed was indicated and complementary treatment was proposed with the Platelet-Rich Fibrin (PRF) technique and topical use of ozonized oil after extraction in the alveolus 48 compared with the technique conventional in alveolus 38. Symptomatic and clinical improvements were observed in region 48 compared to region 38 after 7 days of surgery. Fibrin-rich plasma associated with topical use of ozonized oil proved to be effective in this case of signs and symptoms after surgery and further studies should be carried out to create a clinical treatment protocol.

Keywords: Ozone; Cicatrization; Fibrina rica en plaquetas; Postoperative.

\section{Resumen}

Los terceros molares suelen ser los últimos dientes en erupcionar en la cavidad bucal, en los que hay evidencia de ausencia de espacios en las arcadas dentales, lo que comúnmente los lleva a encontrarse en posición como impactados y / o impactados. En estos casos son frecuentes algunas complicaciones postoperatorias, con presencia de signos y síntomas, como dolor, edema, trismo, alveolitis, hematomas locales y sangrado. Este artículo tiene como objetivo reportar un caso en la literatura de tratamientos complementarios con fibrina rica en plaquetas (PRF) y aceite ozonizado en la extracción de terceros molares en comparación con la técnica convencional. Se realizó una búsqueda de artículos científicos en las bases de datos PubMed y SciELO para la base científica y construcción del reporte de caso clínico. Paciente de sexo masculino, 20 años, con síntoma principal de dolor en los terceros molares inferiores. Tras anamnesis, exploración clínica y radiográfica, se indicó extracción de los dientes 38 y 48 semicerrados y se propuso tratamiento complementario con la técnica de Fibrina Rica en Plaquetas (PRF) y uso tópico de aceite ozonizado tras la extracción en el alvéolo 48 en comparación con la técnica. convencional en el alvéolo 38 . Se observaron mejorías sintomáticas y clínicas en la región 48 en comparación con la región 38 después de 7 días de cirugía. El plasma rico en fibrina asociado con el uso tópico de aceite ozonizado demostró ser efectivo en este caso de signos y síntomas después de la cirugía y se deben realizar más estudios para crear un protocolo de tratamiento clínico.

Palabras clave: Ozono; Curación; Fibrina rica en plaquetas; Postoperatorio.

\section{Introdução}

A extração do terceiro molar (siso) é a intervenção cirúrgica mais executada entre os especialistas em Cirurgia e Traumatologia Buco-Maxilo-Facial (Oliveira, 2006). Os terceiros molares normalmente são os últimos dentes a irromper na cavidade bucal, na qual evidência ausências de espaços nas arcadas dentarias, levando-os comumente a serem encontrados em posição como inclusos e/ou impactados (Dos Santos, 2021).

Atualmente um dos grandes desafios da inquirição clínica prende-se com o desenvolvimento e técnicas complementares bioativas que possam auxiliar no controle dos sinais e sintomas dos pacientes (Dohan, 2006). De acordo com os dizeres de Chiapasco e colaboradores (1993) as cirurgias dos terceiros molares (sisos) estão associadas a experiências desagradáveis dos pacientes, nesses casos são frequentes algumas complicações designadas de morbidade pós-operatória.

Pode-se ter presença de sinais e sintomas de menor complexidade sendo, dor, edema, trismo, alveolite, hematomas locais, sangramentos e de maior complexidade como comunicação buco sinusal, infecções nos espaços faciais, dano ao Nervo Alveolar Inferior, dano a dentes adjacentes, agravamentos periodontais em dentes adjacentes, movimentação dental para áreas nobres da arcada dentária, fratura óssea do tubérculo mandibular e/ou maxilar (Goldberg, Nemarich \& Marco 2nd, 1985; Oliveira, 2006).

A Fibrina Rica em Plaquetas foi descoberto por Dr. Joseph Choukroun para aplicação em cirurgia oral e maxilofacial (Mariano, 2019). A Fibrina Rica em Plaquetas (PRF) é lograda por um método bastante comum, este método embasa na aquisição de amostras do sangue do próprio paciente. Logo após faz-se a centrifugação aonde se obtém uma membrana de fibrina, rica em leucócitos e fatores de crescimentos (PRF). A fibrina libera fatores de crescimento em um período de até 4 semanas (Choukroun et al., 2006).

$\mathrm{Na}$ literatura há indícios da Fibrina Rica em Plaquetas utilizado na atenuação da dor, edema, sangramento, tem o potencial para acelerar a regeneração de tecidos moles e no remodelamento ósseo (Choukroun et al., 2006; Conceição, 2020; Mateus, 2020;). 
O ozônio é um forte oxidante, que se apresenta na forma de oxigênio trivalente (O3) O ozônio tem uma alta capacidade de desinfecção podendo matar bactérias, vírus e esporos de maneira eficaz em poucos segundos (Beretta \& Federici-Canova, F., 2017).

Os estudos mostram que a ozonioterapia é apta e reduz a sensibilidade após cirurgias, pois o ozônio age na assepsia e/ou hemostasia em feridas, como agente irrigador, a terapia também evidência melhorias no processo reparador, pois fomentaram melhor angiogênese e oxigenação local. A técnica também é utilizada no tratamento de quadros inflamatórios. A ozonioterapia acelera a taxa de cicatrização, tornando eficaz tratamento da alveolite e reduz o tempo de cicatrização pósextração formando uma pseudomembrana sobre o alvéolo e abstendo de quaisquer agravos físicos e mecânicos (Nascimento, 2019).

Cada vez os profissionais estão procurando tratamento diferencial, seu valor está em trazer uma melhoria na qualidade de vida nos pós-procedimento de seus pacientes. Perante essas circunstâncias, a demanda por práticas terapêuticas que valoriza a integridade e as formas naturais está sendo explorado (Almeida, Werkman \& Canettieri, 2006).

A associação das duas terapias alternativas devido aos seus benefícios no meio da saúde bucal. Tanto a técnica PRF quanto a ozonioterapia parecem ser efetivas na regeneração e cicatrização dos tecidos lesionados durante o procedimento cirúrgico, auxiliando no controle sintomatológico dos pacientes trazendo uma melhor recuperação no pós-operatório de terceiros molares. Desta forma esse trabalho objetivou em relatar um caso clínico no qual foi comparado a utilização da ozonioterapia e Fibrina Rica em Plaquetas com o tratamento convencional das complicações pós exodontia de terceiros molares.

\section{Metodologia}

A pesquisa que se pretende realizar tem por finalidade analisar sobre a contribuição associada das técnicas de PRF e Ozônio com a técnica convencional. Trata-se de uma pesquisa qualitativa, do tipo exploratória. Quanto aos meios a pesquisa se caracteriza como bibliográfica. A pesquisa bibliográfica foi realizada visando maior compreensão das diferentes interfaces do assunto em questão, através de material acessível ao público em geral, tais como livros, artigos científicos e teses que tratam do tema (GIL, 2002; Lakatos \& Marconi, 2017; Vergara, 2010; Yin, 2001).

Trata-se de um relato de caso de um paciente jovem que possuía os 2 dos sisos semi-erupcionados, escolhido por conveniência do pesquisador e através de aceitação dos respondentes. Este paciente voluntário do sexo masculino, maior de idade aceitou participar do relato de caso e assinou o Termo de Consentimento Livre e Esclarecido (TCLE). O projeto de pesquisa foi aprovado pelo Comitê de Ética em Pesquisa da Faculdade Patos de Minas.

Foi realiza cirurgia para exodontia dos dentes 38 e 48, onde primeiramente foi extraído um dos terceiros molares incluindo as terapias adicionais (PRF e Ozonioterapia, dente 48) e posteriormente foi extraído o outro dente de forma convencional (dente 38).

\section{Relato de Caso}

Foi realizado atendimento clínico com paciente H.D.M, sexo masculino, feoderma, 20 anos, ASA I, que compareceu à Policlínica da Faculdade Patos de Minas (FPM), com queixa principal de sintomatologia dolorosa em região dos terceiros molares inferiores.

$\mathrm{Na}$ anamnese não foi relatado nenhuma doença sistêmica que levasse à contra-indicação da cirurgia. Após análise clínico-radiográfica constatou-se os dentes 38 e 48 semi-inclusos com pericoronarite, falta de espaço na arcada dentaria, curvatura significante da raiz mesial. (Figura 1). 
Figura 1 - Panorâmica Inicial onde mostra a posição dos dentes sisos 38 e 48.

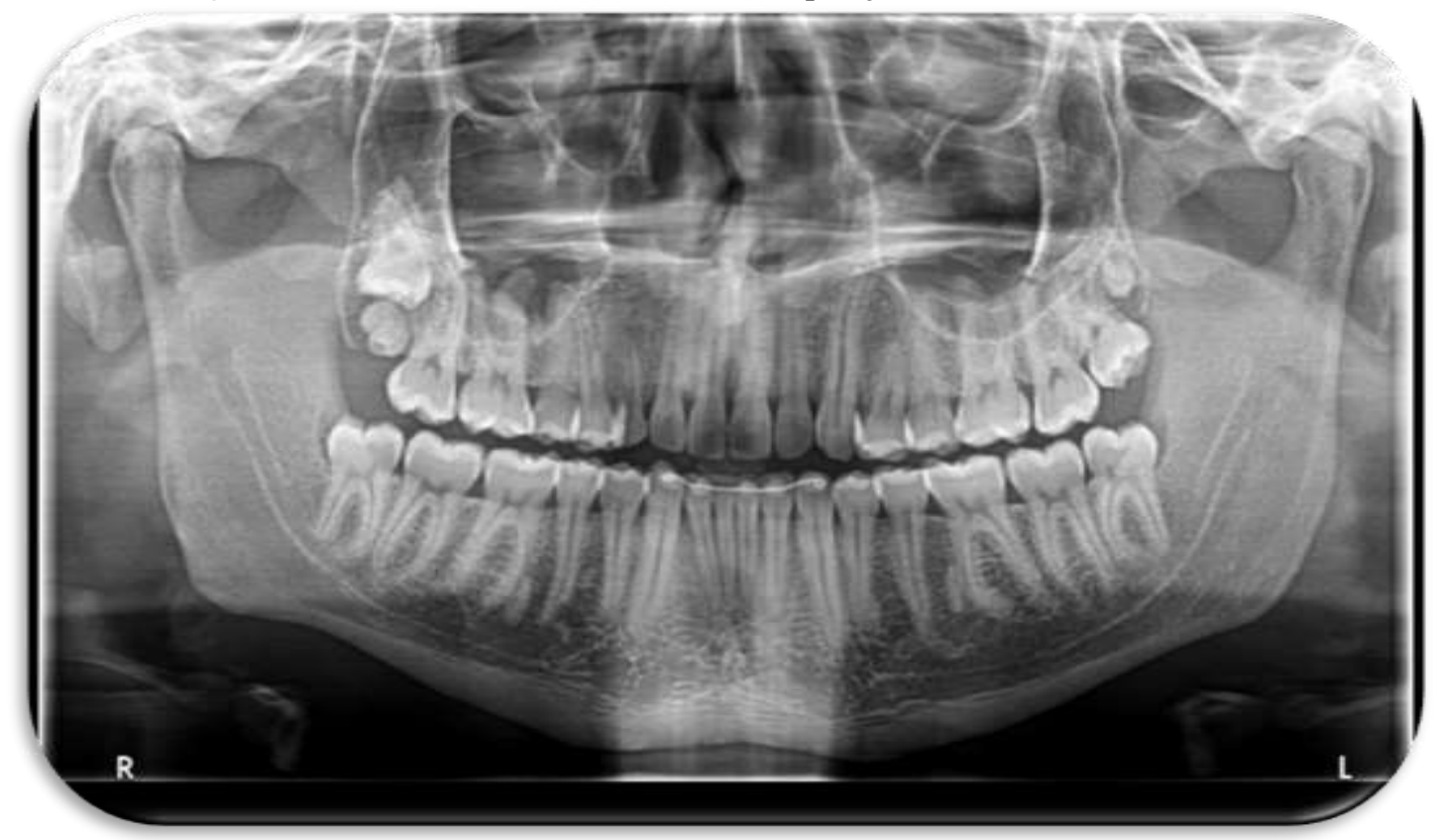

Fonte: Autores (2021).

Logo, foi proposta exodontia dos dois terceiros molares inferiores no planejamento cirúrgico com odontosecção e terapia complementar de enxerto com Fibrina Rica em Plaquetas e uso tópico de óleo ozonizado no dente 48.

Inicialmente foi coletado sangue da veia do paciente para realização do PRF, em seguida a paramentação da equipe, paciente e montagem de mesa clínica, iniciou-se a antissepsia intra-oral utilizando solução de digluconato de clorexidina a $0,12 \%$ e extra-oral solução de digluconato de clorexidina a $2 \%$.

Através da técnica pterigomandibular foram anestesiados os nervos lingual, bucal e alveolar inferior do lado direito e esquerdo da face, onde foi utilizado o anestésico lidocaína à 2\% associado ao vasoconstrictor epinefrina 1:100000.

Foi executada incisão relaxante em envelope em região mesial do segundo molar e distal do terceiro molar com lâmina número $15 \mathrm{C}$, e em seguida descolamento total do retalho gengival para exposição do tecido ósseo com auxílio do descolador de Molt número 9 (Figura 2).

Figura 2 - Descolamento do retalho em volta do dente.

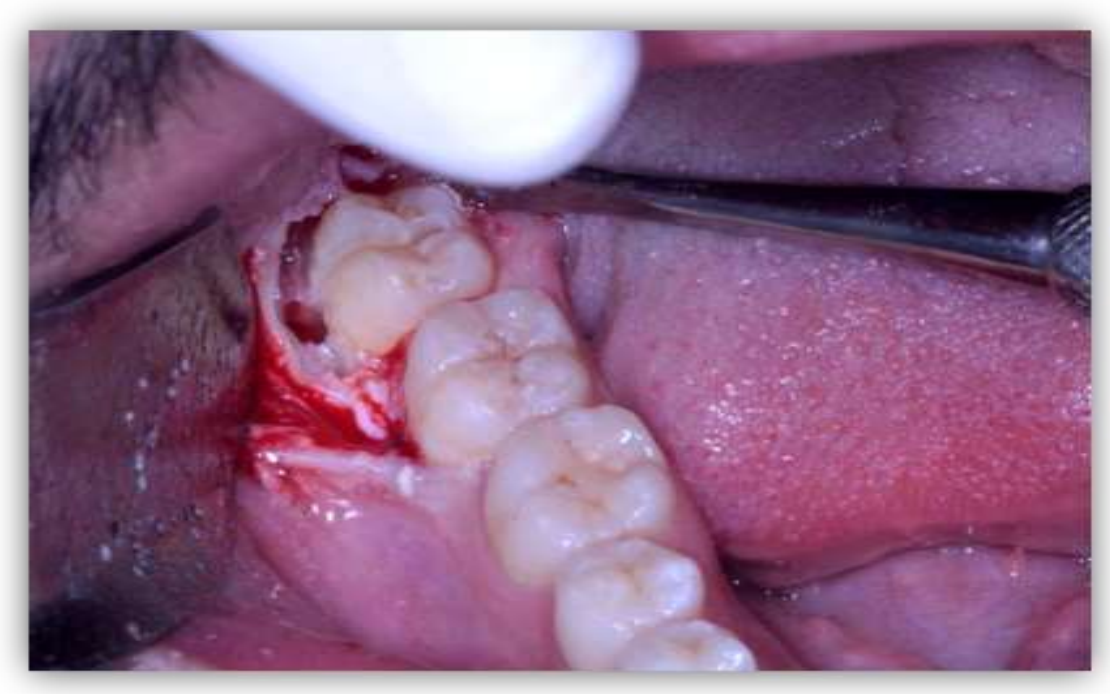

Fonte: Autores (2021). 
Em seguida foi realizada odontosecção no sentido vestíbulo-lingual com broca Zecrya em alta rotação e irrigação com soro fisiológico para separar as raízes (Figura 3).

Figura 3 - Odontosecção com alta rotação utilizando broca carbide 701.

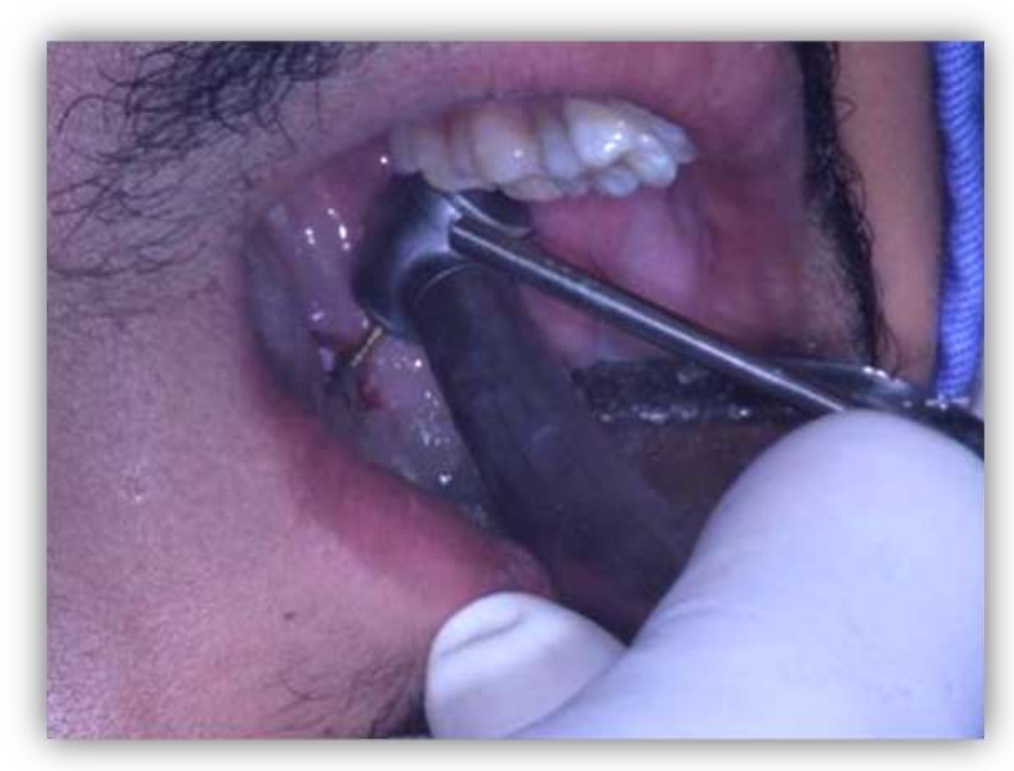

Fonte: Autores (2021).

Para remoção da unidade do alvéolo foi utilizada alavanca reta (Figura 4).

Figura 4 - Remoção do dente de dentro do alvéolo.

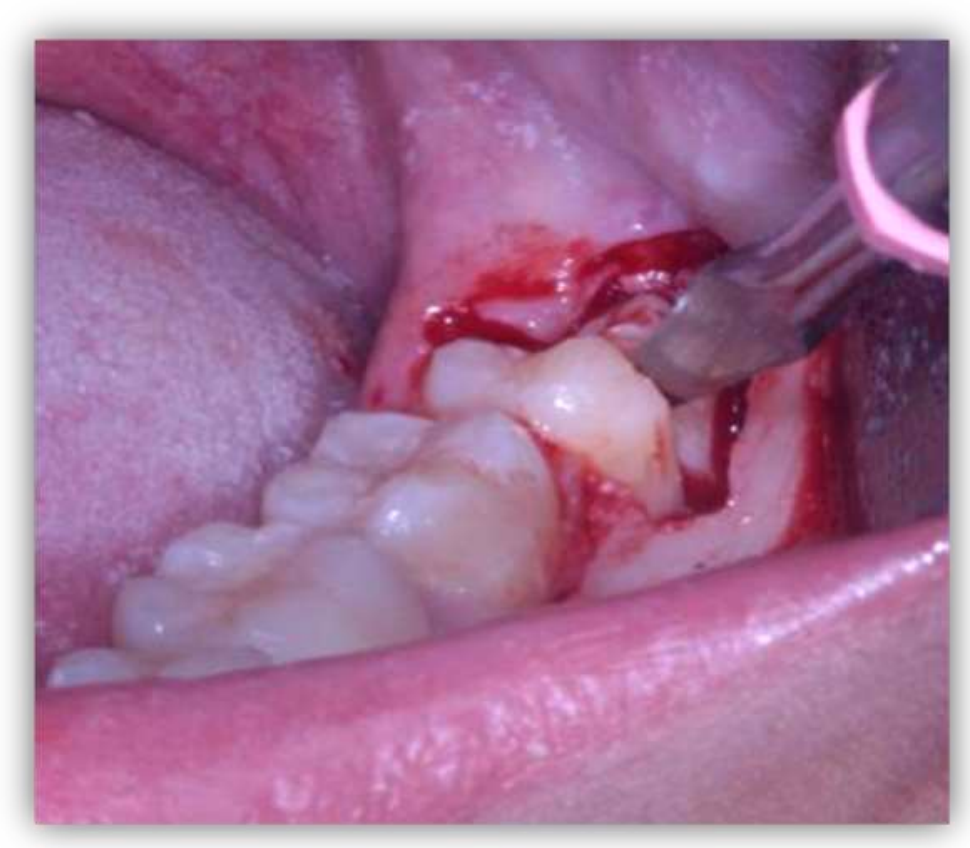

Fonte: Autores (2021)

Após exodontia os cuidados foram tomados (Figura 5), no alvéolo do dente 48 foi realizado o enxerto com PRF 
Research, Society and Development, v. 10, n. 15, e180101522881, 2021

(CC BY 4.0) | ISSN 2525-3409 | DOI: http://dx.doi.org/10.33448/rsd-v10i15.22881

dentro do alvéolo (Figura 6) e a cirurgia concluída através de sutura com pontos simples (Figura 7).

Figura 5 - Coágulo formado dentro do alvéolo após exodontia.

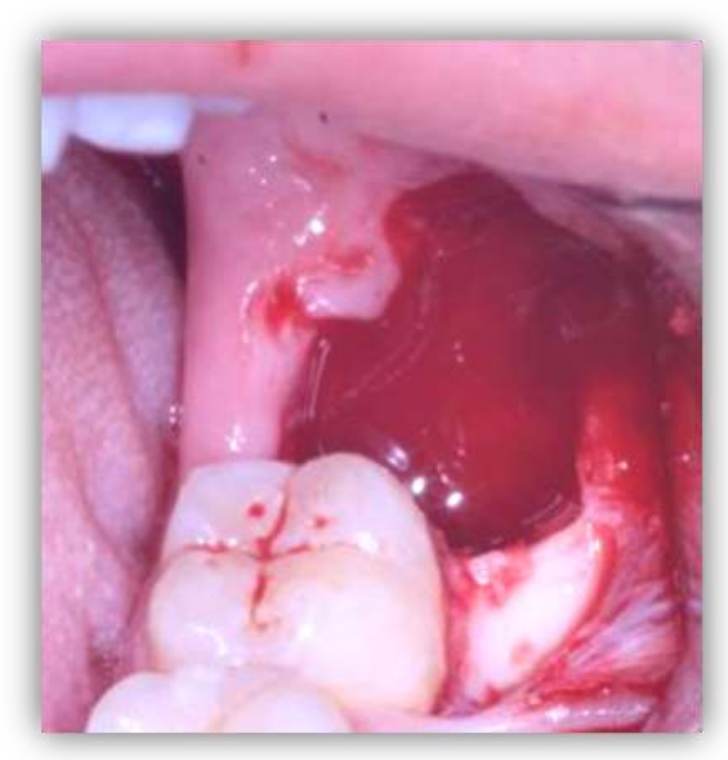

Fonte: Autores (2021).

Figura 6 - PRF dentro do alvéolo.

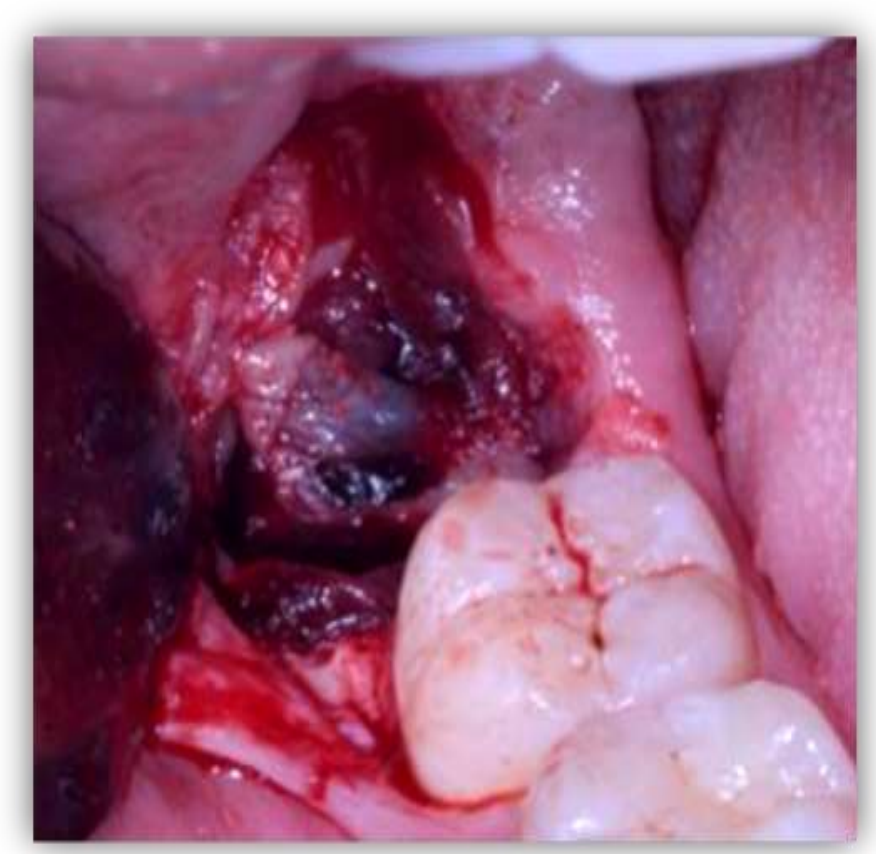

Fonte: Autores (2021). 
Figura 7 - Sutura.

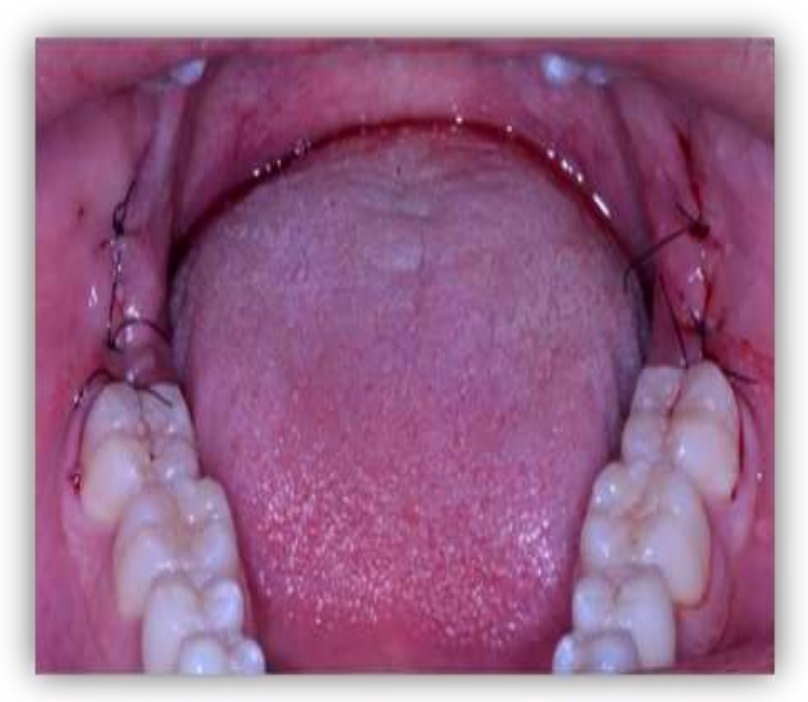

Fonte: Autores (2021).

O paciente foi orientado em utilizar o óleo ozonizado 3 vezes ao dia na região de extração do dente 48 durante 7 dias.

O paciente foi medicado com Amoxicilina 500mg por 7 dias, Ibuprofeno 600mg por 3 dias e Dipirona 500 mg por 3 dias além de ter sido devidamente orientado acerca das instruções pós-operatórias.

Foi realizado o acompanhamento do paciente, através de consulta agendada sete dias após a cirurgia em questão para remoção de sutura (Figuras 8 e 9). Foi observado bom aspecto pós-operatório, com ausência de inflamação, supuração e dor na região do dente 48 que foi tratada com PRF e óleo ozonizado a cicatrização da região do dente 48 foi bem satisfatória quando comparada com a região do dente 38 que teve tratamento convencional apresentando clinicamente com bordas um pouco afastadas (deiscência) e tendo uma boa visualização do alvéolo e tecidos circunscritos em processo de cicatrização, o paciente não apresentou nenhuma queixa que saia da normalidade de um pós-operatório consideravelmente tranquilo. Foi realizado tomada radiográfica panorâmica para acompanhamento (Figura 10). O caso encontra-se em proservação.

Figura 8 - Acompanhamento 7 dias.

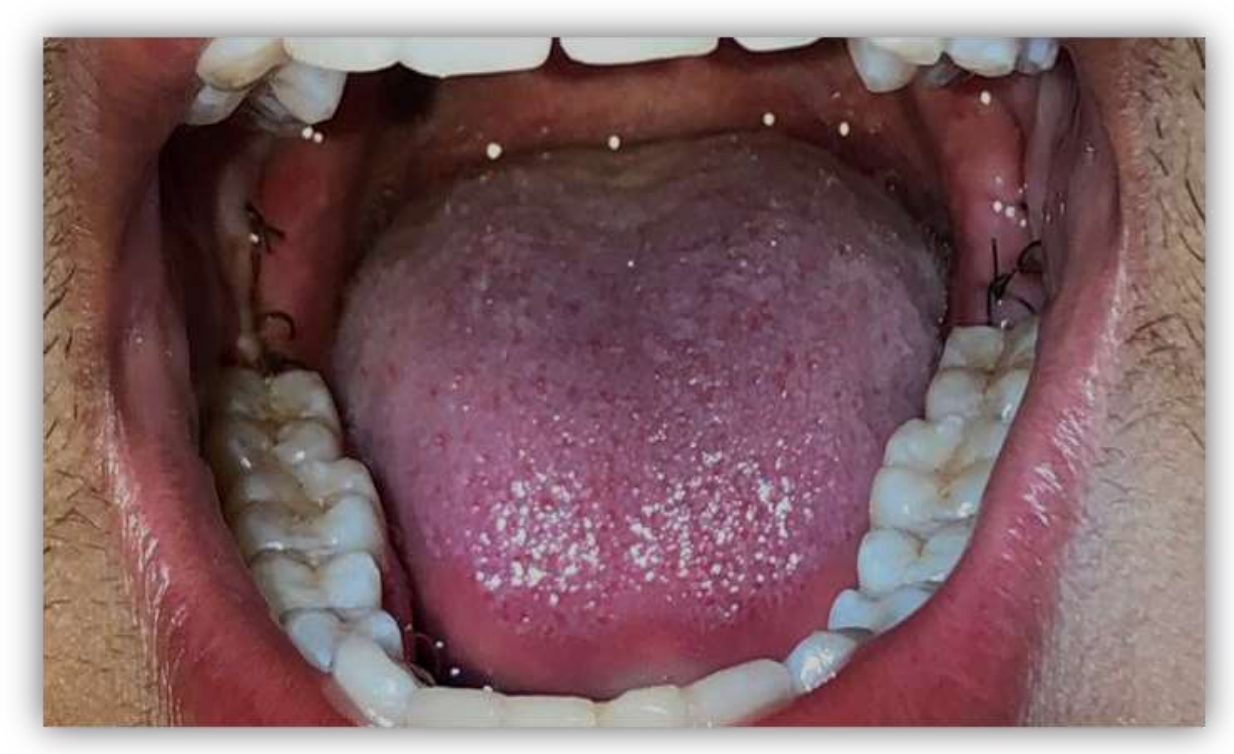

Fonte: Autores (2021). 
Figura 9 - Acompanhamento 7 dias, A- região dente 48 B - região dente 38

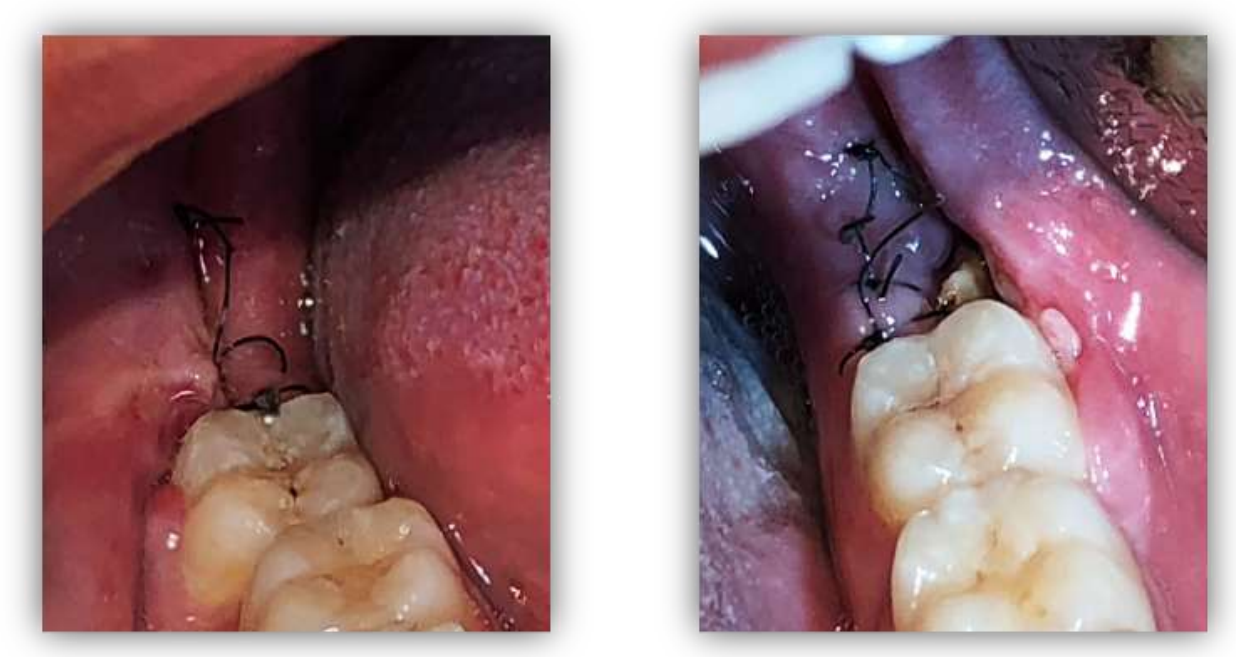

Fonte: Autores (2021).

Figura 10 - Panorâmica de acompanhamento.

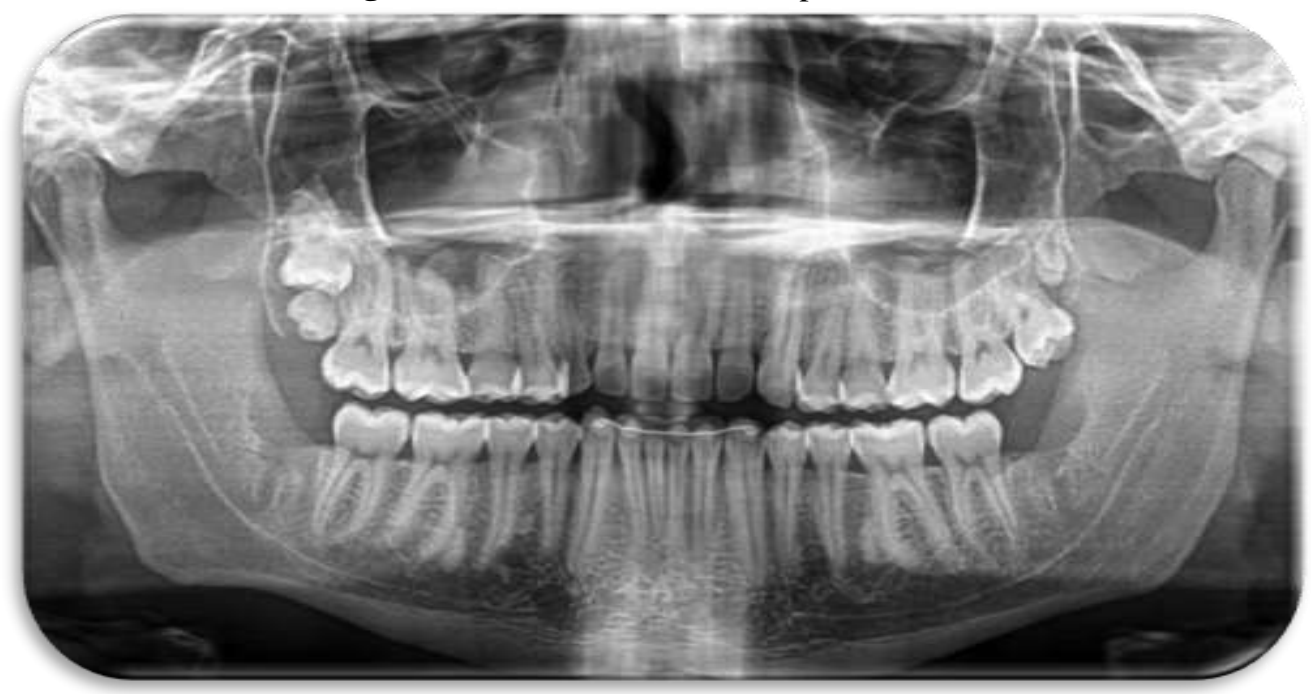

Fonte: Autores (2021).

\section{Discussão}

Quando se trata de complicações relacionadas às exodontias, as mais esperadas variam de hemorragia, dor, trismo e edema até complicações alveolares, existem várias formas de controlar bem essas sequelas, mas métodos alternativos ainda estão sendo estudados e / ou aprimorados para melhorar a qualidade de vida e o tempo de descanso após a cirurgia (Ness, 2016). Neste trabalho o paciente selecionado era saudável, com indicação de extração dos terceiros molares inferiores bilateralmente utilizando técnicas de tratamentos alternativos diferentes para ambos os lados onde não apresentou quaisquer complicações adversas, a região do dente 48 onde foi enxertado PRF e uso tópico de óleo ozonizado o paciente relatou menor dor e incômodo do que a região do dente 38.

Existem vários benefícios clínicos no uso de PRF na prática diária em clínicas odontológicas, desde materiais de preenchimento simples até finas membranas de proteção e revestimentos de enxerto. O custo técnico de obtenção deste material é baixo, pois além de ser um elemento autólogo biologicamente ativo com efeito efetivo de aceleração da cicatrização também pode ser produzido em grandes quantidades como até 8 membranas por vez. (Choukroun et al., 2006). No caso especifico o custo para aplicação da técnica foi realmente baixo, clinicamente o paciente apresentou a área enxertada com a 
aceleração da cicatrização significante comparando com o lado onde o tratamento da extração foi convencional com apenas 7 dias.

Tempo de operação, manejo de tecidos moles e ressecção de tecido ósseo na extração de terceiros molares inclusos podem causar edemas (Hupp, 2009). Por outro lado, ainda existem discussões e divergências na literatura a respeito do A-PRF e sua eficácia na redução do edema (Cardoso, 2012). Compreende-se que quando há maiores dificuldades operatórias, maior será os danos celulares, e assim terá um maior edema. Momentos após a cirurgia já se notou aparição do edema de ambos os lados da face, mesmo sendo dentes semi-inclusos teve suas dificuldades cirúrgicas, o paciente compareceu a Policlínica da FPM dentre 7, 14 e 21 dias após a extração e com auxílio de uma fita métrica medimos ambos os lados da face do paciente, e com 14 dias mostrou um aumento significativo do seu lado direito da face comparado com 7 dias de cirurgia. $\mathrm{O}$ paciente relatou estar se alimento com uma alimentação fora de sua rotina o que poderia ter causado ganha de peso e aumento das bochechas. Deixando a entender um resultado insignificante do PRF referente à diminuição do edema.

A aplicação tópica de óleo ozonizado em cirurgias de tecidos moles foi comprovada perante sua eficiência. O óleo ozonizado acelerou o processo de cicatrização das lesões cirúrgicas em tecido mole a nível epitelial em comparação com outras técnicas (Ferreira et al., 2020). Estudos mostram que o uso tópico de ozônio tem sido eficaz no tratamento de infecções bacterianas, virais e fúngicas, inflamações, grandes feridas, lesões com isquemias entre outras afecções, ele também desempenha uma potente assepsia. Além disso, o óleo ozonizado proporciona estabilidade adequada, o que não acontece em meio aquoso. (Anzolin Da Silveira-Kaross \& Bertol., 2020). No caso especifico dos tecidos na região do dente 48 que foi extraído, o paciente fez o uso do óleo ozonizado 3 vezes ao dia durante 7 dias, o mesmo relatou ter tido um menor incomodo desse local referente a cicatrização e dor quando comparado com a região do 38. Vale ressaltar que a cicatrização e a ausência de dor podem ser provenientes do tratamento em conjunto do PRF e óleo ozonizado, não foi feito uma análise isolada do efeito de cada um. Portanto, acreditamos que o óleo ozonizado pode ter ajudado na desinfecção local e na produção de células epiteliais de cicatrização levando clinicamente um melhor aspecto pós-cirúrgico comparado à região 38.

\section{Conclusão}

Neste caso clínico específico foi observado melhoras sintomatológicas e clínicas na região do 48 comparado com a região do 38 após 7 dias de cirurgia. A Fibrina Rica em Plaquetas associado com uso tópico do óleo ozonizado mostrou-se eficaz neste caso de sinais e sintomas pós cirúrgicos e novos estudos devem ser realizados para a criação de um protocolo clínico de tratamento.

\section{Referências}

Almeida, A. D., Werkman, C. \& Canettieri, A. C. V. (2006). Uso de terapias alternativas no consultório odontológico: uma revisão da literatura. Encontro Latino-Americano De Iniciação Científica, 10. http://www.inicepg.univap.br/cd/INIC_2006/inic/inic/03/INIC0000948.pdf

Anzolin, A. P, Da Silveira-Kaross, N. L. \& Bertol, C. D. (2020). Ozonated oil in wound healing: what has already been proven? Medical gas research, 10(1), 54. https://www.ncbi.nlm.nih.gov/pmc/articles/PMC7871935/

Beretta, M. \& Federici-Canova, F. (2017). A new method for deep caries treatment in primaryteethusing ozone: a retrospectivestudy. Eur J PaediatrDent, 18(2), 111-115. https://ejpd.eu/virtual/download/EJPD_2017_2_4.pdf

Cardoso, M. L. (2012). Fibrina Rica Em Plaquetas E Leucócitos (L-Prf). Diminuindo A Morbidade Em Procedimentos de Reconstruções Teciduais Orais. 2015. 38 f. TCC (Graduação) - Curso de Odontologia, Universidade Federal Fluminense, Nova Friburgo. http://www.punf.uff.br/arquivos_p unf/tcc/od ontologia/2015/1/fibrinaricaemplaquetaseleucocitoslpfrdiminuindoamorbidadeem.pdf

Chiapasco, M., De Cicco, L. \& Marrone, G. (1993) Sideeffectsandcomplicationsassociatedwiththird molar surgery. Oral surgery, oral medicine, oral pathology, 76(4), 412-420. https://sci-hub.se/https://doi.org/10.1016/0030-4220(93)90005-O

Choukroun, J. et al. (2006). Platelet-richfibrin (PRF): a second-generationplateletconcentrate. Part IV: clinicaleffectsontissuehealing. Oral Surgery, Oral Medicine, Oral Pathology, Oral Radiology, andEndodontology, 101(3), e56-e60. https://sci-hub.se/https://doi.org/10.1016/0278-2391(95)90486-7 
Research, Society and Development, v. 10, n. 15, e180101522881, 2021

(CC BY 4.0) | ISSN 2525-3409 | DOI: http://dx.doi.org/10.33448/rsd-v10i15.22881

Conceição, V. S. et al. (2020). Aplicação Da Fibrina Rica Em Plaquetas Em Cirurgia De Terceiros Molares: Avaliação Da Dor (Parte Ii) Application Of Platelet-Rich Fibrin On Third Molar Surgery. Pain Evaluation (Part II). Revista da Faculdade de Odontologia da UFBA, 50(2). https://periodicos.ufba.br/index.php/revfo/article/view/37110/21164

Dohan, D. M. et al. (2006). Platelet-richfibrin (PRF): a second-generationplateletconcentrate. Part I: technologicalconceptsandevolution. Oral Surgery, Oral Medicine, Oral Pathology, Oral Radiology, andEndodontology, 101(3), e37-e44. https://doi.org/10.1016/j.tripleo.2005.07.008

Dos Santos, Y. C. M. et al. (2021). Prevalência de patologias associadas a terceiros molares inclusos: revisão de literatura. Archives Of Health Investigation, 10(2), 351-355. https://www.archhealthinvestigation.com.br/ArcHI/article/download/4695/7049

Ferreira Filho, M. J. S. et al. (2020). A utilização do óleo ozonizado no processo de cicatrização pós cirurgia de implante dental imediato-revisão de literatura. Brazilian Journal of Development, 6(11), 93559-93567. file:///C:/Users/Claudio/Downloads/20772-53330-1-PB.pdf

Gil, A C (2002). Como elaborar projetos de pesquisa. (4a ed.), Atlas.

Goldberg, M. H., Nemarich, A. N. \& Marco 2ND, W. P. (1985). Complicationsafter mandibular third molar surgery: a statisticalanalysisof 500 consecutive procedures in privatepractice. Journalofthe American Dental Association, 111(2), 277-279. https://jada.ada.org/article/S0002-8177(85)12021-0/pdf

Hupp, J. R. (2009). Cirurgia oral e maxilofacial contemporânea. (5a ed.), Elsevier.

Lakatos, E. M. \& Marconi, M. A. (2017). Metodologia científica. (7a ed.), Atlas.

Mariano, Y. C. R. (2019). Exodontia de terceiro molar com o uso fibrina rica em plaquetas avançada: relato de caso clínico. http://131.0.244.66:8082/jspui/bitstream/123456789/1654/1/Exodontia\%20de\%20terceiro\%20molar\%20com\%20o\%20uso\%20de\%20fibrinarica\%20em\%20pl aquetas\%20avan\%C3\%A7ada\%20\%282\%29.pdf

Mateus, D. C. B. (2020). Avaliação clínica da influência da aplicação da membrana de L-PRF na cicatrização após exodontia de terceiros molares inclusos e semi inclusos. Tese de Doutorado. https://comum.rcaap.pt/bitstream/10400.26/35039/1/Mateus_Dina_do_Carmo_Batista.pdf

Nascimento, M. (2019). Ozonioterapia na odontologia. Brasil Escola UOL. https://repositorio.ufu.br/handle/123456789/29237

Ness, G. M. et al. (2016). Princípios de Cirurgia Bucomaxilofacial de Peterson. (3a ed.), Santos, 31(9), 85-96. http://famamportal.com.br:8082/jspu i/bitstream/123 456789/1654/1/Exodontia\%20de\%20terceiro\%20molar\%20com\%20o\%20uso\%20de\%20fibrinarica\%20em\%20plaquetas\%20avan\%C3\%A7a da\% $20 \% 282 \% 29$.pdf

Oliveira, L. B. de et al. (2006). Avaliação dos acidentes e complicações associados à exodontia dos 3os molares. Rev. cir. traumatol. buco-maxilo-fac, 51-56. https://www.revistacirurgiabmf.com/2006/v6n2/v6n27.pdf

Vergara, S. C. (2010). Projetos e Relatórios de Pesquisa em Administração. (12a ed.), Atlas.

Yin, R. K. (2001). Estudo de caso: planejamento e métodos. (2a ed.), Bookman. 